\title{
The Best of Many Worlds, or, is Quantum Decoherence the Manifestation of a Disposition?*
}

\author{
Florian J. Boge
}

\begin{abstract}
In this paper I investigate whether the phenomenon of quantum decoherence, the vanishing of interference and detectable entanglement on quantum systems in virtue of interactions with the environment, can be understood as the manifestation of a disposition. I will highlight the advantages of this approach as a realist interpretation of the quantum formalism, and demonstrate how such an approach can benefit from advances in the metaphysics of dispositions. I will also confront some commonalities with and differences to the many worlds interpretation, and address the difficulties induced by quantum non-locality. I conclude that there are ways to deal with these issues and that the proposal hence is an avenue worth pursuing.
\end{abstract}

Keywords: quantum decoherence • propensity interpretation of quantum theory • mutual manifestation $\cdot$ continuous manifestation

\section{Introduction: The Decoherence Mechanism and the Problem of its Interpretation}

In quantum theory (QT), the state of a system $S$ is famously described by a (time-dependent) state vector $|\mathcal{S}(t)\rangle$, an element of a suitable complex vector space $\mathcal{H}$. Vectors can be superposed to give new vectors; $|S(t)\rangle$ might have an expansion as $\alpha_{1}(t)\left|S_{O_{1}}\right\rangle+\alpha_{2}(t)\left|S_{o_{2}}\right\rangle+\ldots=$ $\sum_{j} \alpha_{i}(t)\left|S_{o_{j}}\right\rangle$, and the $\left|S_{o_{k}}\right\rangle$ might be eigenstates of some observable (measurable quantity) $O$, i.e. states in which $O$ is standardly taken to assume definite value $o_{k}$ on $S$.

Quantum superposition is the origin of all troubles arising in the interpretation of the formalism: It figures in interference phenomena, non-commutativity of observables, entanglement, and the non-classical probabilistic structure of the theory. Worse: allowing that some

${ }^{*}$ This is a pre-print version of an article accepted by Studies in History and Philosophy of Modern Physics. The accepted version includes minor changes. 
macroscopic measuring device $\mathcal{M}$ could be described by QT as well (why would it not, if all its elementary components can?), the joint evolution of $\mathcal{M}$ and $S$ would formally proceed as

$$
|S(t)\rangle\left|\mathcal{M}_{0}(t)\right\rangle=\sum_{j} \alpha_{j}(t)\left|S_{j}\right\rangle\left|\mathcal{M}_{0}(t)\right\rangle \mapsto \sum_{j} \tilde{\alpha}_{j}\left(t^{\prime}\right)\left|S_{j}\right\rangle\left|\mathcal{M}_{j}\right\rangle,
$$

where $t<t^{\prime},\left|\mathcal{M}_{0}(t)\right\rangle$ is a 'ready state', and typically $\tilde{\alpha}_{j}\left(t^{\prime}\right) \neq \alpha_{j}\left(t^{\prime}\right)$. This is one version of the so called measurement problem, as $\mathcal{M}$ is now entangled with $S$ and superposed w.r.t. a range of possible measurement outcomes.

Decoherence theory puts a stop to at least part of the madness. Assuming that the environment $\mathcal{E}$ is in some state $\left|\mathcal{E}_{0}\right\rangle$, the joint evolution of $\mathcal{M}, S$, and $\mathcal{E}$ from $t^{\prime}$ to $t^{\prime \prime}>t^{\prime}$ is

$$
\sum_{j} \tilde{\alpha}_{j}\left(t^{\prime}\right)\left|S_{j}\right\rangle\left|\mathcal{M}_{j}\right\rangle\left|\mathcal{E}_{0}\left(t^{\prime}\right)\right\rangle \mapsto \sum_{j} \underbrace{\tilde{\tilde{\alpha}}_{j}\left(t^{\prime \prime}\right)}_{=: \beta_{j}\left(t^{\prime \prime}\right)}\left|S_{j}\right\rangle\left|\mathcal{M}_{j}\right\rangle\left|\mathcal{E}_{j}\right\rangle .
$$

Writing this in the density matrix-formalism (and renaming $t^{\prime \prime} \mapsto t$ ), one can now eliminate $\mathcal{E}$ again to obtain an effective state for $\mathcal{S}$ and $\mathcal{M}$ only:

$$
\begin{aligned}
\hat{\rho}_{S \mathcal{M}} & =\operatorname{tr}_{\mathcal{E}} \hat{\rho}_{S \mathcal{M} \mathcal{E}}=\sum_{i, j} \beta_{i} \beta_{j}^{*}\left|\mathcal{S}_{i} \times \mathcal{S}_{j}\right| \otimes\left|\mathcal{M}_{i} \times \mathcal{M}_{j}\right|\left\langle\mathcal{E}_{j} \mid \mathcal{E}_{i}\right\rangle_{t} \\
& \stackrel{t \mapsto t+\delta}{\approx} \sum_{j}\left|\beta_{j}\right|^{2}\left|S_{j} \times S_{j}\right| \otimes\left|\mathcal{M}_{j} \times \mathcal{M}_{j}\right|
\end{aligned}
$$

where the approximation holds after a short time span $(\delta)$ if one can justify that $\left\langle\mathcal{E}_{j} \mid \mathcal{E}_{i}\right\rangle_{t} \rightarrow 0$ for $i \neq j$ as time increases. This is possible for scenarios ranging from scattering to magnetization, and has lead to the testable and tested prediction of decoherence times (cf. Joos et al., 2003; Schlosshauer, 2007).

Decoherence has a bunch of merits: it singles out a preferred basis of the joint space $\mathcal{H}_{S} \otimes \mathcal{H}_{\mathcal{M}}$, and thereby eliminates the so called preferred basis problem (e.g. Schlosshauer, 2007, p. 54), the problem that even a joint state of $S$ and $\mathcal{M}$ remains ambiguous due to the possibility of basis changes. Assuming, moreover, that the $\left|S_{k} \times S_{k}\right|$ decompose some (coarse) observable ${ }^{1}$ $O=\sum_{j} o_{j}\left|S_{j} \times S_{j}\right|$ and the $\left|\mathcal{M}_{k} \times \mathcal{M}_{k}\right|$ some pointer observable $X=\sum_{j} x_{j}\left|\mathcal{M}_{j} \times \mathcal{M}_{j}\right|$, these observables will be stable under the continuing interaction with the environment, and in this sense be preferred as well (e.g. Schlosshauer, 2007, p. 77). Most importantly, 'any interaction described by a potential $V(r)$ is diagonal in position, hence position is always the distinguished "observable" measured by the interaction [...].' (Joos et al., 2003, p. 55; original emphasis) But the localization thus achieved will usually be sufficiently coarse so as to also allow for the simultaneous ascription of coarse momenta. ${ }^{2}$ Hence coarse positions and momenta are usually preferred observables, and in this sense, decoherence may be said to "recover classicality" (cf. Joos et al. 2003, pp. 64 ff.; Schlosshauer 2007, pp. 115 ff.).

Decoherence also justifies talk of an "effective collapse" to the extent that it corresponds to a

\footnotetext{
${ }^{1}$ For simplicity I allow to sometimes merge operators and observables notationally, while they should of course be strictly separated conceptually.

${ }^{2}$ Recall that position and momentum are non-commuting observables, and that a position eigenstate $|x\rangle$ has an expansion proportional to $\int \mathrm{d} p e^{-i p x}|p\rangle$, whence it is a superposition of momentum eigenstates.
} 
practically irreversible delocalization of phase relations into the composite systemenvironment state induced by inevitable and ubiquitous environmental monitoring [...] [and] leads to effectively nonunitary dynamics for the local system that may manifest themselves (for example) in the decay of interference patterns [...]. (Schlosshauer, 2007, p. 69; first emphases added, second in original)

Inquiring on small fragments of the environment (e.g. the laboratory equipment) of a decohered state, one will quickly obtain virtually all available information about the system, whereas for other, random quantum states, this does not hold (cf. Zurek, 2009). In this sense, decoherence 'mimics' the dynamics of a collapse mechanism.

There are, however, some serious drawbacks. The result in (1), for instance, looks like a statistical mixture, which could be interpreted as an expression of ignorance about the joint system's true quantum state $\left|S_{j} \times S_{j}\right| \otimes\left|\mathcal{M}_{j} \times \mathcal{M}_{j}\right|$. But this mixture is improper, as it arises by neglect of $\mathcal{E}$, not by statistical weighting. And in principle, observables can be defined which would expose differences between the two in the long-run (cf. d'Espagnat, 1990, p. 1154 ff.). There is, moreover, the possibility of recoherence in (1), i.e. of the reoccurrence of measurable interference, which can nicely be demonstrated on systems like atoms in a cavity (cf. Schlosshauer, 2007, p. 257 and references therein).

So we cannot interpret $\hat{\rho}_{S \mathcal{M}}$ as an expression of ignorance, and we do not retrieve one (joint) state for $S$ and $\mathcal{M}$ with definite values for $O$ and $X$ respectively from the decoherence process. The following comment by Bell (1990, p. 25) hits the mark:

The idea that elimination of coherence, in one way or another, implies the replacement of "and" by "or", is a very common one among solvers of the "measurement problem". It has always puzzled me.

Decoherence also does not fit nicely with most interpretations of QT (such as collapse models or Bohmian theories) that manage to replace the "and" by an "or" by other means (cf. Schlosshauer, 2004, pp. $1291 \mathrm{ff}$.). The currently most popular way to circumvent the problem is the many worlds interpretation (MWI), which loosely says that the "and" persists, and that decoherence merely defines different branches of a "multiverse", that will afterwards be more or less independent (e.g. Saunders et al., 2010; Wallace, 2012).

The MWI, however, still faces a probability problem, as it is difficult to reconcile with the probabilistic content of QT, i.e. Born's rule, stating that the probability of measuring $o_{k}$ for $O$ on $S$ in state $|S(t)\rangle=\sum_{j} \alpha_{i}(t)\left|S_{o_{j}}\right\rangle$ at time $t$ is $\left|\alpha_{j}(t)\right|^{2}$. Approaches that appeal to decoherence to derive the Born rule are threatened by circularity (cf. Baker, 2007), and rely on axioms that are hard to defend (cf. Maudlin 2014, p. 804; Dizadji-Bahmani 2013; ref. blinded, pp. 259-60). Other approaches assume a well defined number of branches (e.g. Zurek, 2005; Carroll and Sebens, 2014), which seems incompatible with the decoherence mechanism and the standard probability calculus (cf. Wallace, 2012, p. 120).

Another alternative are "anti-realist" interpretations like Healey's pragmatist approach, according to which 'decoherence involves a physical process', but 'a system's quantum state' does not 'describe or represent properties of systems involved in such a process.' (Healey, 2012, p. 1538; original emphasis) Healey, however, remains vague on issues like the process involved in but not described by decoherence, questions of semantics, or what "physical reality" even 
means, given that quantum theory is not descriptive, and that we also lack other means for a fundamental description (cf. Bell, 1964; Pusey et al., 2012; Colbeck and Renner, 2012; Hardy, 2013).

There seem to be three principled options: (a) go into an even more anti-realist direction than Healey and answer all questions by recursion to epistemological concerns alone (as suggested e.g. in ref. blinded, ch. 5), (b) advance a satisfying account of the quantum probabilities within the MWI, or (c) reconcile decoherence with some realist alternative to the MWI. In this paper I am going to pursue the third option, along a path that today has become rather marginal in the debate. ${ }^{3}$

CAVEAT: My approach will be quite 'Viking', in the sense of French (2014), meaning that I "ruthlessly plunder" the metaphysics of dispositions-debate without respecting boundaries (of worked-out metaphysical positions), and without necessarily giving anything back.

\section{Decoherence as Manifestation}

\subsection{From Heisenberg's Potentialities to the Metaphysics of Dispositions}

The locus classicus of allusions to modal notions in the interpretation of QT are certainly the philosophical writings of Heisenberg. He in particular claimed that if one considers the word "state" as describing some potentiality rather than a reality [...] then the concept of "coexistent potentialities" is quite plausible,' and that 'the atoms or the elementary particles themselves [...] form a world of potentialities or possibilities rather than one of things or facts.' (Heisenberg, 1958, pp. 159-60; emphasis added) He also believed that the Born probabilities expressed 'statements about possibilities or better tendencies ("potentia" in Aristotelian philosphy), and these statements are completely objective, they do not depend on any observer[...].' (Heisenberg, 1958, p. 53) As Friederich (2013, p. 94) points out, this 'is very much in spirit with what is nowadays called the "propensity interpretation" of probability', most commonly associated with Popper (1957).

Like Heisenberg, Popper $(1990,1957)$ uses the term "tendencies", but there are discernible differences between both positions: In his early works, Popper (1957, p. 68; emphasis omitted) believed that 'the propensity interpretation [...] takes the mystery out of quantum theory, while leaving probability and indeterminism in it.' He compared the change of the state vector in a double slit experiment with one slit closed to 'the shifting of a pin on a pin board' meaning that '[e]very change in the experimental arrangement such as the shutting of a slit, will lead to a different distribution of weights to the possibilities[...].' (Popper, 1957, p. 69; emphasis omitted) And he also 'stressed that the propensities are not only as objective as the experimental arrangements but also physically real-in the sense in which forces, and fields of forces, are physically real.' (ibid.; original emphasis)

Heisenberg (1958, p. 54), in contrast, spoke of a "transition from the "possible" to the "actual"' that 'takes place as soon as the interaction of the object with the measuring device, and thereby with the rest of the world, has come into play[...]'; and he found that 'physicists have gradually

\footnotetext{
${ }^{3}$ Of course there are appeals to dispositions in parts of the debate (e.g. Dorato and Esfeld, 2010; Esfeld et al., 2014; Egg and Esfeld, 2015), but these are typically formulated against the background of another realist interpretation such as Bohmian Mechanics or some objective collapse model.
} 
become accustomed to considering the electronic orbits, etc., not as reality but rather as a kind of "potentia".' (Heisenberg, 1958, p. 156; empahsis added)

Popper, in fact, also radically changed his understanding of propensities over time: 'Popper's later view[...] on propensity[...] retains from the earlier Popper objective singular probabilities, but abandons the association of propensities with repeatable conditions. Instead propensities are associated with states of the universe.' (Gillies, 2000, p. 822) In 1990, Popper expressed the conviction 'that propensities should not be regarded as properties inherent in an object, such as a die or a penny, but that they should be regarded as inherent in a situation [...].' (Popper, 1990, p. 14) This is to be contrasted, e.g., with von Mises (1957, p. 12; emphasis added), who allowed that ' $[\mathrm{t}]$ he probability of a 6 is a physical property of a given die and is a property analogous to its mass, specific heat, or electrical resistance.'

This change in Popper's views was not without consequence: his allusions to the solution of all of QT's mysteries by propensities are quite faint in his 1990, and they play a subordinate role therein. In particular, the mentions of QT here almost exclusively concern issues of determinism (cf. pp. 15-7). Suárez (2009, pp. 503-4), moreover, recovers 'three lethal objections' form the literature, 'that render it [Popper's interpretation at any time - FJB] untenable.' In short they are that (i) Popper cannot properly distinguish superpositions and mixed states since he identifies propensities with probabilities, that he (ii) cannot really account for interference after all, and that (iii) propensities are time-asymmetric while (the relevant) conditional probabilities are not, whence the identification of propensities and probabilities becomes problematic in the first place.

Heisenberg (1958, p. 53), on the other hand, allowed that a probability assignment in QT also 'contains statements about our knowledge of the system, which of course are subjective in so far as they may be different for different observers.' Because of this 'omelette that nobody has seen how to unscramble' (Jaynes, 1990, p. 381), Friederich (2013, p. 87; original emphasis), for instance, reads Heisenberg as an 'opportunistic thinker, whose remarks on the interpretation of quantum mechanics are mainly motivated by dialectical purposes and do not lead to a coherent foundational perspective.' From neither Popper's nor Heisenberg's thinking hence emerges a view of propensities that allows for a clear understanding of the quantum formalism, useful for solving the measurement problem.

Margenau (1954) probably came the farthest in advancing such a view. ${ }^{4}$ He suggested to distinguish between

possessed and latent observables. Possessed are those, like mass and charge of an electron, whose values are "intrinsic," do not vary except in a continuous manner, as for example the mass does with changing velocity. The others are quantized, have eigenvalues, are subject to the uncertainty principle, manifest themselves as clearly present only upon measurement. I believe they are "not always there," that they take on values when an act of measurement, a perception, forces them out of indiscriminacy or latency. (Margenau, 1954, p. 270; original emphasis)

So like von Mises, Margenau is willing to locate propensities in the objects, not experimental setups or the entire universe. But he relies exclusively on measurement - or even perception -

${ }^{4}$ Cf. also Suárez (2007, pp. 419 ff.) for an appraisal. 
to carry the 'latency' over into definiteness. His views thus fall prey to standard problems, like Wigner's friend (Wigner, 1961, p. 873). ${ }^{5}$

Propensities have been identified as a specific kind of dispositions (cf. Popper 1957, pp. 67 and 68; Mumford 1998, p. 11; Ellis 2001, p. 129), dispositions which are not 'sure fire' (Mumford, 1998, ibid.) but rather 'stochastic' (Ellis, 2001, ibid.; emphasis omitted). Dispositions, in turn, are characterized as properties that manifest themselves only under appropriate stimulus conditions (e.g. Mumford 1998, pp. 6 ff.; Molnar 2003, p. 84). As is well known, the precise logical analysis of this natural language description is a troubling endeavor (e.g. Mumford 1998, pp. $36 \mathrm{ff}$.; Martin 2008, pp. $12 \mathrm{ff}$.). But the intuitive level suffices to make some sense in the quantum context.

As a first step, we can use it to invalidate Popper's claims to the need of involving an entire experimental setup or even the rest of the universe in the ascription of propensities. Popper (1990, p. 15), namely,

pointed out that the propensity of a penny to fall on a flat table with heads up is obviously modified if the table top is appropriately slotted. Similarly, one and the same loaded die will have different propensities if the table top is very elastic rather than of marble, or if it is covered by a layer of sand.

Now a penny will have some tendency to land heads, some tendency to land tails, and some tendency to land on its side in circumstances with or without slots on the table. Clearly, these tendencies will not be identical for both cases. But does that mean that the dispositions of the penny themselves, i.e. its very properties which make it so that it stochastically exhibits one of these behaviors, are changed as well? As can be seen, I am inclined, against Popper and like Margenau or von Mises, to view propensities as properties of the object in question alone. Then in this sense, the propensities of the penny could be said to remain entirely invariant under the change of the table, while their tendency to manifest would be altered, resulting in the different observed frequencies in both setups. In other words: the stimulus conditions figure in the determination of the strengths of an object's tendencies to manifest certain propensities, but they do not alter the propensities themselves.

I hence suggest some non-standard terminology here, ${ }^{6}$ thereby separating the two notions (tendency and propensity) in meaning. This not only avoids Popper's problematic confusion of propensities and probabilities, but also fits more naturally with a realistic reading of the quantum formalism in terms of dispositions, as we shall see.

\subsection{Mutual Manifestation}

Equation (1) left us with something akin to a statistical mixture of states, which, however, could not be interpreted directly in terms of ignorance. I want to suggest that the traditional proposals about propensities figuring in the interpretation of QT, together with some advances

\footnotetext{
${ }^{5}$ Remarkably even Wigner, referring back to Zeh's seminal (1970) paper on decoherence, later wrote: 'For some time I believed that the mere phenomenon of the observation of the outcomes of measurements shows [...] [that there are] phenomena which are still outside of physics' interest[.] This has been at least superficially eliminated by Zeh's observation[...].' (Wigner, 1986, p. 379)

${ }^{6}$ E.g. Vetter (2015, pp. 63 ff.) for a similar move w.r.t. potentialities and dispositions.
} 
in the metaphysics of dispositions, can be put in the service of understanding decoherence as an "effective collapse theory".

The basic proposal is simple: A system $S$ 's loss of interference due to decoherence, together with the empirical fact that only one value, o, out of a range of possible values for observable $O$ is observed on $S$ in a given experiment, somehow corresponds to the manifestation of a disposition, the disposition to exhibit $O(S) \approx o$ under suitable environmental conditions, with frequencies predicted by the Born rule. This would acknowledge the central role of the Born probabilities right away, make our non-acquaintance with macroscopic superpositions understandable, and remove the "ontological excess baggage"7 of multiple, more or less independent "branches". But how exactly should this be understood?

Before I go into the intricacies, let me first draw attention to a feature of the entangling interaction leading to decoherence that can be understood quite well by appeal to a dispositionalist lingo. A standard conviction in the decoherence literature is that the reason that the environment ${ }^{8}$ appears to us entirely "classical" lies in the fact that the systems within it will mutually decohere each other:

For example, the gravitational interaction between the earth and the moon leads rapidly to mutual decoherence. Similarly, a charge can show only a limited coherence range in the presence of other charges [...]. (Joos et al., 2003, p. 55; emphasis added)

The reason is clear enough: the symmetry properties of an entangled state of multiple interacting (elementary) systems allow to single out any one of them as the system of interest, and to observe, by partial tracing, the vanishing of coherence on $i$.

A similar kind of reciprocity has been acknowledged the metaphysics of dispositions: C. B. Martin, in particular, suggests to think of an object with a certain disposition and the appropriate stimulus conditions as 'reciprocal disposition partners for a mutual manifestation[...].' (Martin, 2008, p. 3; emphasis added) While Martin's own writings are sometimes a bit opaque, the idea is nicely illustrated by Mumford and Anjum (2011, p. 34) as follows:

Instead of thinking, for instance, of water having a power to dissolve salt, we should think of water and salt as mutual manifestation partners whose manifestation is the production of saline solution. And two objects manifest their gravitational mass - a disposition to attract - only in tandem.

Not only is salt soluble, but water also dissolving. Viewing both as 'reciprocal disposition partners' and the stimulus conditions as mutual, both these intuitions can be captured at the same time.

That the situation is remarkably similar for decohering interactions can be illustrated on a simple model of spins decohered by an environment of other spins (Zurek, 1982). ${ }^{9}$ The Hamil-

\footnotetext{
${ }^{7}$ I misappropriate the term from Hardy (2004).

${ }^{8}$ Above I have talked, as is standard practice, of a threefold distinction between systems involved in the decoherence process: some microscopic system $\mathcal{S}$, a macroscopic measuring device $\mathcal{M}$, and the environment $\mathcal{E}$. Clearly, $\mathcal{M}$ could be construed as part of $\mathcal{E}$, as $\mathcal{E}$ will (usually) contain all sorts of macroscopic systems. I will hence largely omit talk of a measuring device below.

${ }^{9}$ I here closely follow Schlosshauer's (2007, pp. 89 ff.) exposition.
} 
tonian of the interaction is here given as

$$
\hat{H}_{\text {int }}=\frac{1}{2} \hat{\sigma}_{z}^{(0)} \otimes \sum_{i=1}^{N} \mu_{i} \hat{\sigma}_{z}^{(i)} \bigotimes_{j \neq i} \mathbb{1}_{j}=\hat{H}_{\mathrm{int}_{S}} \otimes \hat{H}_{\mathrm{int}_{\mathcal{E}}},
$$

where $\hat{\sigma}_{z}^{(k)}$ is the Pauli- $z$-martix acting on the space of the $k$ th system, $\mathbb{1}_{k}$ is the identity operator acting on that space, and the $\mu_{k}$ are interaction constants. Eigenstates of $\hat{H}_{\text {int }}$ will be product states of spins up or down along the $z$-axis, like $|n\rangle=\left|\uparrow_{1}\right\rangle\left|\downarrow_{2}\right\rangle \ldots\left|\downarrow_{N}\right\rangle$, and the associated energies will be $\epsilon_{n}=\sum_{j=1}^{N}(-1)^{m_{j}} \mu_{j}$, where $m_{k}=0$ if spin $k$ is up along $z$ and 1 if it is down. Simple combinatorics tells us that there will be $2^{N}$ possible such states, so we can let $n$ range from 1 to $2^{N}$.

Assuming that $S$ is initially in a superposition $|\mathcal{S}(0)\rangle=\alpha\left|\uparrow_{0}\right\rangle+\beta\left|\downarrow_{0}\right\rangle$ and $\mathcal{E}$ in $|\mathcal{E}(0)\rangle=$ $\sum_{n=1}^{2^{N}} \gamma_{n}|n\rangle$, the joint evolution given by $\hat{U}(t)=\exp \left(-\frac{l}{\hbar} \hat{H}_{\text {int }} t\right)$ will effect a coupling $\alpha\left|\uparrow_{0}\right\rangle\left|\mathcal{E}_{1}(t)\right\rangle+$ $\beta\left|\downarrow_{0}\right\rangle\left|\mathcal{E}_{2}(t)\right\rangle$, where

$$
\left|\mathcal{E}_{1}(t)\right\rangle=\sum_{n=1}^{2^{N}} \gamma_{n} e^{-\frac{l}{2 \hbar} \epsilon_{n} t}|n\rangle, \quad\left|\mathcal{E}_{2}(t)\right\rangle=\sum_{n=1}^{2^{N}} \gamma_{n} e^{\frac{l}{2 \hbar} \epsilon_{n} t}|n\rangle .
$$

Tracing out the environment leads to the effective density matrix

$$
\hat{\rho}_{S}(t)=|\alpha|^{2}\left|\uparrow_{0} X \uparrow_{0}\right|+|\beta|^{2}\left|\downarrow_{0} X \downarrow_{0}\right|+\alpha \beta^{*} d(t)\left|\uparrow_{0} X \downarrow_{0}\right|+\alpha^{*} \beta d^{*}(t)\left|\downarrow_{0} X \uparrow_{0}\right|,
$$

where $d(t)=\left\langle\mathcal{E}_{1}(t) \mid \mathcal{E}_{2}(t)\right\rangle=\sum_{n=1}^{2^{N}}\left|\gamma_{n}\right|^{2} e^{-\frac{l}{\hbar} \epsilon_{n} t}$ is sometimes called a decoherence factor. Since at any $t, d(t)$ corresponds to the addition of $2^{N}$ vectors of lengths $\left|\gamma_{n}\right|^{2}$ rotating in the complex plane with frequencies $\epsilon_{n} / \hbar$, the analysis of $d(t)$ can be understood in terms of a twodimensional random walk, where direction and length of the step are given by orientation and length of the vectors at $t$ respectively. Since $\sum_{n=1}^{2^{N}}\left|\gamma_{n}\right|^{2}=1$, the average step length will be $2^{-N}$ from which it follows that $\langle|d(t)|\rangle \propto 2^{-N}$. It can be shown, furthermore, that for a broad range of distributions of the $\mu_{i}$ and $N$ large enough $d(t) \approx e^{-\Gamma^{2} t^{2}}$, where $\Gamma^{2}$ depends on the initial state and the distribution of the $\mu_{i}$ (cf. Schlosshauer, 2007, pp. 91-2 and references therein for the details). In other words: for large enough $N \hat{\rho}_{S}(t)$ will approximate a mixture of states $\left|\uparrow_{0} \times \uparrow_{0}\right|$ and $\left|\downarrow_{0} X \downarrow_{0}\right|$ with weights $|\alpha|^{2}$ and $|\beta|^{2}$ as $t$ increases. This is decoherence in action.

But the crucial thing to realize, for the present purposes, is that the relevant states $\left|\mathcal{E}_{1}(t)\right\rangle,\left|\mathcal{E}_{2}(t)\right\rangle$ from which the decohering behavior can be derived develop in virtue of the interaction of $\mathcal{E}$ with $S$. In other words: there will be a change in the environmental spins, $\mathcal{E}$, in the presence of the additional spin, $S$, and that change of $\mathcal{E}$ in virtue of the interaction with $S$ is essential for decoherence to take place. Hence, just as salt and water mutually manifest their solving and dissolving behaviors to give rise to a solvent do single and environmental spins mutually manifest their decohered and decohering behaviors.

\subsection{No "or" form an "and": Continuous Manifestation and the Removal of Collapse}

Appeal to mutual manifestation gives first credence to decoherence having to do with the manifestation of dispositions. But it clearly does not solve the central problem of any realist interpretation of QT: the measurement problem. In $|S(0)\rangle=\alpha\left|\uparrow_{0}\right\rangle+\beta\left|\downarrow_{0}\right\rangle$, $S$ has a disposition 
to spin along the $z$ axis, which could manifest as spinning-up along $z$ or as spinning-down along $z$ (it is not sure fire). And $S$ will manifest its $z$-spinning behavior under suitable circumstances, such as $\left|\mathcal{E}_{1}(t)\right\rangle$ and $\left|\mathcal{E}_{2}(t)\right\rangle$, as $\left|\uparrow_{0}\right\rangle$ and $\left|\downarrow_{0}\right\rangle$ respectively. ${ }^{10}$ But the entangled state $\alpha\left|\uparrow_{0}\right\rangle\left|\mathcal{E}_{1}(t)\right\rangle+\beta\left|\downarrow_{0}\right\rangle\left|\mathcal{E}_{2}(t)\right\rangle$ still has both situations occur. So according to the quantum formalism (read dispositionally), both these dispositions will manifest at the same time. This is problematic, as we retrieve nothing but a many worlds-story with a dispositionalist touch, and all the problems associated with probability remain.

What is still missing here is 'the selection step' (Fuchs and Schack, 2012, p. 245), the “"selection" of one of the entangled state's components' which 'singles out a particular measurement result.' (ibid.) Simply adding a spontaneous 'jump' into one of the multiple possibilities amounts to nothing but the plain old, minimalist Dirac-von Neumann interpretation, which raises all sorts of further questions (e.g. Bub, 1997, pp. 31-2). The question is whether a dispositionalist account can somehow "fake", and hence do away with the need for a proper selection step. I think that the answer is "yes", but it requires some effort.

Now recall, first of all, that while decoherence does not lead to a proper collapse, it at least selects a preferred basis and thereby reduces the system's "dispositional freedom", if you will. Since basically any old interaction will induce such reductions, this leads me to the following "first axiom" for a dispositionalist analysis:

$\left(\mathrm{DA}_{1}\right) \quad$ Only perfectly isolated systems instantiate collections of entirely nonmanifest dispositions. Any interaction will impinge on the non-manifestness. Ambiguity due to the possibility of basis changes indicates that the system has dispositions w.r.t. values of all measurable quantities, even non-comeasurable ones.

I should explain a little. Take the prime example of non-comeasurable observables, position $x$ and momentum $p$, with commutator $[x, p]=-\imath \hbar$. This commutator leads to the infamous Heisenberg uncertainty relation $\Delta x \Delta p \geq \frac{\hbar}{2}$, connected to the fact that any more precise position measurement cannot reveal much about momentum, and vice versa. A free, spinless system will have a state with position representation $\psi(\boldsymbol{x})=\int \mathrm{d}^{3} p \tilde{\psi}(\boldsymbol{p}) e^{\frac{l}{\hbar} \boldsymbol{x} \boldsymbol{p}}$, where the momentum representation $\tilde{\psi}(\boldsymbol{p})$ is given, by Fourier transformation (which amounts to a "basis change"), as $\tilde{\psi}(\boldsymbol{p})=\int \mathrm{d}^{3} x \psi(\boldsymbol{x}) e^{-\frac{l}{\hbar} \boldsymbol{x} \boldsymbol{p}}$. On the dispositionalist account, we can be flesh this out to mean that the system has propensities for showing up in any volume in the support of $\psi(\boldsymbol{x})$ and for exhibiting any momentum that lies in the support of $\tilde{\psi}(\boldsymbol{p})$, with tendencies of strengths $|\psi(\boldsymbol{x})|^{2} \mathrm{~d}^{3} x$ and $|\tilde{\psi}(\boldsymbol{p})|^{2} \mathrm{~d}^{3} p$ respectively; but that no precise values of localization and momentum will ever manifest simultaneously in any measurement or like situation.

In the context of decoherence, the most important application of spatial wavefunctions are certainly scattering scenarios (e.g. Schlosshauer, 2007, pp. $119 \mathrm{ff}$.). Here the dynamics of a density matrix $\rho\left(\boldsymbol{x}^{\prime}, \boldsymbol{x}\right)=\left\langle\boldsymbol{x}^{\prime}|\hat{\rho}| \boldsymbol{x}\right\rangle$ (which for a pure state is just $\psi\left(\boldsymbol{x}^{\prime}\right) \psi^{*}(\boldsymbol{x})$ ) is governed by a differential equation that leads to the vanishing of $\rho\left(\boldsymbol{x}^{\prime}, \boldsymbol{x}\right)$ for $\boldsymbol{x}^{\prime} \neq \boldsymbol{x}$, just as $\left|\uparrow_{0} X \downarrow_{0}\right|$, say, will vanish in (4) for $t \longrightarrow \infty$. To appreciate this, let me propose the following "second axiom":

$\left(\mathrm{DA}_{2}\right) \quad$ In decoherence, the stimulus conditions are such that all the dispositions associated with an observable will manifest.

\footnotetext{
${ }^{10}$ In this sense, the propensity to exhibit $z$-spinning behavior could also be thought of as 'multi-track' (cf. Ellis and Lierse, 1994, p. 29).
} 
For both these situations, we hence need to deal with the fact that $\rho\left(\boldsymbol{x}^{\prime}, \boldsymbol{x}\right)$ and $\left|\uparrow_{0} X \downarrow_{0}\right|$ would still be present for $t<\infty$, and with the fact that $\rho\left(\boldsymbol{x}^{\prime}, \boldsymbol{x}^{\prime}\right)$ would still be present in the final state at " $t=\infty$ " even if the system was measured to be at $\boldsymbol{x} \neq \boldsymbol{x}^{\prime}$, as would $\left|\downarrow_{0} X \downarrow_{0}\right|$ in (4) if the system was measured to have spin up.

I think that what Hüttemann (2004, cf. p. 19) calls continuously manifestable dispositions (CMDs) here comes to good use. Hüttemann (ibid.) introduces CMDs to understand how laws of nature can be approximately true in virtue of the manifestations of dispositions. For instance, by a physical law, the specific heat $c_{V}$ of a crystal is given as a function of the phonon density and the temperature, and the law strictly speaking applies to an ideal crystal only. But determining the amount of impurities in some set of samples and then ordering them according to purity one may extrapolate the behavior to infer that in the ideal case the law actually holds. Hence Hüttemann (2004, p. 20; emphasis added) has it 'that the disposition' to exhibit a particular value for the specific heat in accordance with the law 'is partially manifest in the non-ideal situation and that the transition from the less than ideal to the ideal is continuous so as to allow for extrapolation.'

Appeal to CMDs in the analysis of quantum decoherence allows us to remove both the selection step and the many worlds that make a coherent understanding of the quantum probabilities so difficult, along the following lines. As long as the dispositions associated with a system's quantum state are "all on an equal footing", they can properly interfere with one another, in accordance with Heisensberg's thinking. This is especially the case before any decoherence has taken place, in accordance with $\left(\mathrm{DA}_{1}\right)$. When decoherence then takes place, ' $\left.\mathrm{t}\right]$ he environment, composed of many subsystems, acts as an amplifying, higher-order measuring device for the state of the system [...].' (Schlosshauer, 2007, p. 69; emphasis added) This will happen in a continuous fashion, meaning that e.g. the ability of the two states $\left|\uparrow_{0}\right\rangle$ and $\left|\downarrow_{0}\right\rangle$ to interfere with one another is gradually-albeit usually quite quickly-reduced over time. And the effect of this process will usually also depend on some suitable quantity determining the system's ability to interact with the "environment" (like its internal energy; cf. the plots of visibility of "fringes" in Brune et al. 1996, p. 4889 and in Arndt et al. 2001).

Since (selective) measurements on a decohered state will, however, unambiguously indicate one out of the many values the system is disposed to exhibit, only one of these dispositions must have been amplified so much as to become detectable. The remainder, in contrast, can only be present 'latently', to borrow Margenau's term; but it must be still so present, as evidenced by recoherence. Hence thinking, say, of "spin up" and "spin down" along some direction as CMDs, this means that "spin up" might be manifest to a high degree, under suitable environmental conditions, but that "spin down" would be manifest at the same time, albeit to a far lesser degree.

Let us introduce some terminology, to present things more cogently. I suggest to call the disposition which has been amplified so much as to lead to the observable behavior dominant or dominantly manifest. The remainder we may call effectively latent. The crucial thing to realize is that they are all manifest once decoherence has occurred, but not all to the same degree. The gradual vanishing of interference fringes and the possibility of recoherence, which has been experimentally verified even on relatively large objects (e.g. Raimond et al., 1997), urges that this picture is preferred over Margenau's. The Born rule weights then obtain a direct and crucial meaning: they quantify the tendency of each of an observable's values to 
become dominant, so as to determine the system's observable behavior. Decoherence factors and their vanishing, moreover, can then be read as quantifying the "struggle for dominance" in this process, and the fact that the "winner" will not be "bothered by the losers" anymore, after some (short) time.

This leads me to the "third axiom", which removes the tension created by $\mathrm{DA}_{2}$ :

$\left(\mathrm{DA}_{3}\right)$ In the manifestation process represented by the decoherence mechanism, only one out of all the manifesting dispositions will manifest dominantly, and the Born probabilities quantify each disposition's tendency to do so. The remainder will become effectively latent, thereby not contributing to observable behavior. Decoherence factors quantify the tendency for swaps in dominance, which becomes almost negligible for most larger systems after very short times.

Now Hüttemann (2004, p. 21; original emphasis) urges that the use of the word "continuity" [...] is not meant to imply that the manifestation of a disposition is a process that takes some time', since while he does 'not deny that there might be such manifestation processes,' he is 'interested in [...] manifestation as the product of such a process.' These considerations transfer reasonably well to the case of decoherence. Clearly, there are interactions at play in decoherence, whose dynamics is described by a (continuous) unitary evolution. But it is not the continuity of these processes which determines e.g. the 'sharpness' of localization in the scattering case, but rather the number number of scatterings in a certain time interval (cf. Schlosshauer, 2007, p. 127). Thus, the degree of manifestation may vary in a(n almost) continuous fashion in decoherence as well, ${ }^{11}$ and it is the result of a certain (set of) continuous process(es).

In summary, Heisenberg's transition from the possible to the actual is, on the account established, always gradual; and Margenau's step out of latency is never perfect nor at all triggered by conscious observation per se, but rather by physical interactions that stimulate mutual manifestation. Moreover, while the dispositions in question are strictly inherent in objects, not setups, Popper was at least half right in that the precise frequencies deriving from the associated tendencies, and quantified by the Born rule weights, will depend on both system and environment (ultimately the entire universe).

\section{Open Questions}

\subsection{Minds and Worlds}

The foregoing considerations lay a reasonable foundation for an interpretation of decoherence in terms of dispositions. However, the fact that the dispositions involved in some quantum state will all manifest to some degree, on my account, makes it still unpleasantly reminiscent of a

\footnotetext{
${ }^{11}$ The vanishing of interference terms in scattering scenarios is in fact usually described by a differential equation, which indeed suggests a continuous process. The derivation however involves a couple of "tricks" and idealizations such as a move from a discrete to a continuous range of possible scattered momenta (cf. Schlosshauer, 2007, p. 125). That a continuum of degrees of manifestation is established in the sense of interpolation, as with Hüttenann's example of the specific heat, can also be seen from typical plots (e.g. Brune et al., 1996; Arndt et al., 2001).
} 
crude version of the MWI. As in the MWI, the entire content of a decohered state is not merely possible but somewhat actual. And there will be a loss of interference, so that the different dispositions may be said to be "branching", in a loose manner of speaking. But the thing that is missing for my dispositional account to collapse into the MWI is the association of additional consciousnesses with those dispositions that become effectively latent.

To see the point more clearly, let us briefly assess the role of consciousness in the MWI. Maybe the most detailed assessment of this issue is Albert and Loewer (1988, pp. 206-7), who introduced the many minds view of the MWI: 'every observer[...] has associated with it not a single mind but rather an infinite set of minds', and the Born probability, derived from the squared modulus of a coefficient of some state in a superposition, 'is a measure of the "proportion" of minds' associated to that very state.

Now Albert and Loewer's original proposal did not take notice of decoherence yet, but Lockwood (1996, p. 185; original emphasis) believed that the stability of the pointer basis might answer the question ' why consciousness and perception should favour the states that they do.' Zeh (2000, p. 226; original emphasis) similarly writes that when decoherence (and branching) take place,

[i]t is not the real world (described by a wave funtion) [sic] that branches in this picture, but consciousness (or rather the state of its physical carrier), and with it the observed (apparent) "world" [...].

And Wallace (2012, p. 3; original emphasis) equally describes the MWI with decoherence as

both a many-worlds and a many-minds theory, in the sense that it entails that there are a great many versions of myself, living in surroundings much like my own and interacting with other versions of yourself, elsewhere in physical reality.

But there is a certain problem associated with even this many minds view, in the decoherence based version appreciated by Lockwood, Zeh, and Wallace. This problem is that the "branches" of the "mutliverse" are never really separated, but will always (weakly) overlap. The conscious states associated with actual observations, however, do not overlap at all-I simply do not feel to have perceived "spin down" the least bit when my spin measurement clearly reveals "spin up". Consciousness, in other words, will have to "cut off" the branches in a way that decoherence strictly never does.

So states of consciousness will have to be added to the MWI, as they cannot be identical with any of the (always slightly overlapping) physical states, and Albert and Loewer's verdict that 'any many worlds interpretation which respects' that agents can correctly access and report their own beliefs 'will be committed to some form of non-physicalism' (1988, p. 206; original emphasis) seems entirely correct. This invalidates a central virtue of the MWI, 'that it purports to explain how to make sense of quantum theory without adding extra equations or interpretational postulates.' (Kent, 2010, p. 311) But consciousness thus also receives a rather central role for the MWI, as it is only in relation to conscious experience that the "apparent", "non-overlapping" worlds exist; those worlds in which we gather all evidence for QT and decoherence, after all. 


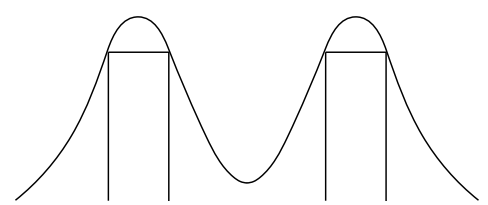

(a) Overlap between different branches vs. non-overlapping states of consciousness in the MWI

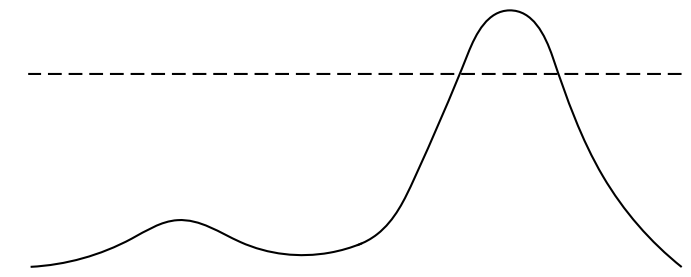

(b) Latently vs. dominantly manifest dispositions

Figure 1: Symbolic illustration of many worlds vs. CMDs: The "box states" in (a) indicate the states of consciousness; the dashed line in (b) indicates the threshold of conscious perception.

Viewed by these lights, the dispositionalist account that I have suggested here thus (somewhat surprisingly) reserves a much less important role for consciousness: our conscious experience does not determine which tendency becomes amplified, it can only be used to determine which tendency was amplified. And over repetitions of similar trials, the rates of such amplifications will be approximately in accordance with the Born probabilities, since these quantify the strength of a given propensity's tendency to become amplified in the given environmental conditions.

Still, my view bears resemblances to what Albert and Loewer (1988) call the single mind view of the MWI; that when an observer $\mathcal{A}$ measures (say) a spinful system in a superposition of spin up and down along some axis with (real) coefficients $c_{1}$ and $c_{2}$ respectively, 'the probability that $\mathcal{A}$ 's mind ends up believing that spin is up $=c_{1}^{2}$ and the probability that $\mathcal{A}$ 's mind ends up believing that spin is down $=c_{2}^{2}$. (Albert and Loewer, 1988, p. 205)

The problems with this single mind view are numerous, for instance that as long as one assumes that 'when $\mathcal{A}$ sincerely reports that she has a definite belief about the value of $x$ $\operatorname{spin}[\ldots]$ then $\mathcal{A}$ does believe that the $x$-spin has a definite value' (Albert and Loewer, 1988, $\mathrm{p}$. 205), it implies that 'mental states do not even supervene on brain states [...] since one cannot tell from the state of a brain what its single mind believes.' (Albert and Loewer, 1988, p. 206; original emphasis) And it equally creates the 'mindless hulk problem', namely that '[i]f I embark on what I take to be a conversation with my wife, how would I know, on this view, that there was really “anyone at home"?' (Lockwood, 1996, p. 175; original emphasis)

My dispositionalist assessment of decoherence is different in that I have assumed only one of the dispositions to become amplified over the others for all conscious observers. In this way, consciousness may supervene on the brain states (it is associated only with the dominant ones), there will be no mindless hulks (every conscious biological organism has a conscious mind, associated with its dominantly manifest dispositions), and, as I have explained, consciousness does not play any fundamental role in the interpretation of the theory.

Somewhat of a weak spot remains in this connection though: nothing in the formalism besides maybe the very existence of different amplitudes and their successful interpretation in terms of probability - really tells us that one of the dispositions will be amplified over the 
others. That I gather from experience alone, which similarly serves as a motivation for all collapse theories.

\subsection{Spooky Manifestation at a Distance?}

Probably the biggest threat to my proposal is the well-known non-locality issue, which haunts (almost) every realist interpretation of QT. ${ }^{12}$ Take a system of two spins in the singlet state $|\chi\rangle=\frac{1}{\sqrt{2}}\left(\left|\uparrow_{L}\right\rangle\left|\downarrow_{R}\right\rangle-\left|\downarrow_{L}\right\rangle\left|\uparrow_{R}\right\rangle\right)$ and an EPR-Bohm type measurement performed on them (cf. Bohm, 1951, p. $614 \mathrm{ff}$.). The measurement of spin $L$ on the left hand side of the experiment will immediately decohere the state and hence amplify, according to the present analysis, one of the dispositions (spin up, spin down) over the other. But the decohering interaction on spin $L$ will immediately fix the dispositions of spin $R$, even if it is at a spacelike distance and should hence not be causally accessible, at least according to the standard reading of the relativity theories (e.g. Taylor and Wheeler 1963, p. 39; Friedman 1983, p. 159).

To see this sort of "nonlocal influence" in the context of decoherence more clearly, take the entangled singlet state $|\chi\rangle$ again. Assume, further, that the interaction that is used to determine the spin value on either side is of the Zurek-type considered above, but that in the "laboratory frame" it first happens on the left hand side only. ${ }^{13}$ This means that before the interaction, the total state will be

$$
|\Psi\rangle=\frac{1}{\sqrt{2}}\left(\left|\uparrow_{L}\right\rangle\left|\downarrow_{R}\right\rangle-\left|\downarrow_{L}\right\rangle\left|\uparrow_{R}\right\rangle\right)\left|\mathcal{E}_{0, L}\right\rangle\left|\mathcal{E}_{0, R}\right\rangle,
$$

and the Hamiltonian will be of the form

$$
\hat{H}_{\mathrm{int}, R}=\frac{1}{2} \hat{\sigma}_{z} \otimes \mathbb{1}_{S, R} \otimes \sum_{i=1}^{N} \mu_{i} \hat{\sigma}_{z}^{(i)} \bigotimes_{j \neq i} \mathbb{1}_{j} \otimes \mathbb{1}_{\mathcal{E}, R}
$$

with obvious meanings of the symbols. Notably, I have here assumed the two environments on the left and on the right to be factorizing, meaning that they can be thought of as being entirely disjoint. The unitary evolution will then effect the change

$$
\hat{U}|\Psi\rangle=\frac{1}{\sqrt{2}}\left(\left|\uparrow_{L}\right\rangle\left|\downarrow_{R}\right\rangle\left|\mathcal{E}_{1, L}(t)\right\rangle-\left|\downarrow_{L}\right\rangle\left|\uparrow_{R}\right\rangle\left|\mathcal{E}_{2, L}(t)\right\rangle\right)\left|\mathcal{E}_{0, R}\right\rangle,
$$

where $\left|\mathcal{E}_{1, L}(t)\right\rangle$ and $\left|\mathcal{E}_{2, L}(t)\right\rangle$ are defined in analogy to the decohering environmental states in eq. (3). Now since $\left|\mathcal{E}_{0, R}\right\rangle$ remains completely unchanged, tracing out both left and right environments will lead to the state

$$
\begin{aligned}
\hat{\rho}_{S_{L}, S_{R}}(t)=\frac{1}{2}\left|\uparrow_{L} X \uparrow_{L}\right| & \otimes\left|\downarrow_{R} X \downarrow_{R}\right|+\frac{1}{2}\left|\downarrow_{L} X \downarrow_{L}\right| \otimes\left|\uparrow_{R} X \uparrow_{R}\right| \\
& -\frac{1}{2} d(t)\left|\uparrow_{L} X \downarrow_{L}\right| \otimes\left|\downarrow_{R} X \uparrow_{R}\right|-\frac{1}{2} d^{*}(t)\left|\downarrow_{L} X \uparrow_{L}\right| \otimes\left|\uparrow_{R} X \downarrow_{R}\right|,
\end{aligned}
$$

\footnotetext{
${ }^{12}$ I say 'almost' because here the MWI indeed has a major advantage: the genesis of the quantum correlations reduces entirely to the impressions of observers in a "quantum multiverse", in virtue of their local interactions (cf. Albert and Loewer, 1988, p. 210). All of this, of course, notwithstanding the problems of talking about probability (and hence: correlation) and consciousness in the MWI in the first place.

${ }^{13}$ I will also assume the two particles which are entangled in spin-space to be distinguishable, so that I can disregard considerations of entanglement in position space (cf. Ghirardi et al., 2002, p. 63).
} 
which looks almost exactly like the state in (4), but for two correlated systems and with the particular choice $\alpha=-\beta=2^{-1 / 2}$.

If we apply the suggested dispositionalist interpretation and assume that $d(t)$ vanishes sufficiently fast, we have the result that one of the two propensities becomes almost immediately amplified on the right hand side as well, namely that anti-aligned to the one on the right hand side-even though the relevant environments were assumed to be perfectly disjoint. This seems exactly like the causal sort of dependence between the two outcomes that has riddled the debate for so long.

Now a lot has been written on this topic, far too much to be seriously covered in this paper. I will hence indulge in some cherry picking to outline a way to deal with this difficulty in the dispositionalist proposal.

Basically, there are three main options for a causal account of the situation (e.g. Suárez, 2004; Näger, 2016): (i) the outcomes directly influence each other causally, (ii) the distant setting (of the measuring device and environmental conditions) causally influences the local outcome, or (iii) there is a (hidden) common cause for the correlation.

Now as regards options (i) and (ii), a result by Näger (2013) suggests that dependence on both settings needs to be assumed to avoid the derivation of a Bell-type inequality. This is unproblematic in principle for the dispositionalist: The local environment, featuring also the "setting conditions", will obviously figure in the local manifestation; and that the remote environment will apparently exert an influence as well has already been acknowledged. Nevertheless, the causation would be superluminal, and hence imply daunting consequences like the in-principle possibility of causal loops.

Option (iii) has been suggested e.g. by Mittelstaedt (1998) and Näger (2016). While Näger (cf. 2016, p. 1148) appeals to the traditional collapse-vocabulary, ${ }^{14}$ Mittelstaedt (1998, p. 158; emphasis added) allows that 'the environment acts - as a common cause [...] - and provides simultaneously the objectification' of two spin observables' values along the same axis and on opposite arms of the experiment.

Näger (2016, p. 1128; original emphasis), however, points out that 'the measurement statistics of entangled quantum objects (which violate Bell inequalities) is per se in conflict with usual principles of causal explanation', i.e. 'even if one disregards all spatiotemporal constraints[.]' And the environment too is subject to this constraint, when construed as a common cause.

To see this more clearly take, in particular, the time evolved quantum state $\hat{\rho}_{S_{L}, S_{R}}(t)$ in which the environment on the left hand side has exerted its influence, and is assumed to have caused both measurable correlated values. Then we have

$$
\begin{aligned}
& p\left(\uparrow_{L}, \downarrow_{R} \mid \hat{\rho}_{S_{L}, S_{R}}(t)\right)=\operatorname{tr}\left(\left|\uparrow_{L} X \uparrow_{L}\right| \otimes\left|\downarrow_{R} X \downarrow_{R}\right| \hat{\rho}_{S_{L}, S_{R}}(t)\right)=\frac{1}{2}>\frac{1}{4}= \\
& =\operatorname{tr}\left(\left|\uparrow_{L} X \uparrow_{L}\right| \otimes \mathbb{1}_{S, R} \hat{\rho}_{S_{L}, S_{R}}(t)\right) \operatorname{tr}\left(\mathbb{1}_{S, L} \otimes\left|\downarrow_{R} X \downarrow_{R}\right| \hat{\rho}_{S_{L}, S_{R}}(t)\right) \\
& =p\left(\uparrow_{L} \mid \hat{\rho}_{S_{L}, S_{R}}(t)\right) p\left(\downarrow_{R} \mid \hat{\rho}_{S_{L}, S_{R}}(t)\right) .
\end{aligned}
$$

Construed as a common cause of the amplifications, the environment would, in other words, violate Reichenbach's "screening-off"-condition (1965, p. $165 \mathrm{ff}$.), a principle at the heart (in some version or other) of formal theories of causation (e.g. Wroński 2014; Spirtes et al. 2000,

\footnotetext{
${ }^{14}$...which, to recall, may not be compatible with decoherence (cf. Schlosshauer, 2004, pp. 1294 ff.).
} 
p. 29). Long story short: if we think of the relation between the two manifestation events as causal, this would violate the causal constraints implied by the relativity theories and the probabilisitic-causal constraints of standard accounts of probabilistic causation.

There is a recent proposal by Gebharter and Retzlaff (unpublished) that I believe can be exploited to remove the tension within the parameters of my dispositionalist account. Gebharter and Retzlaff, namely, suggest that correlations like these could be understood in virtue of 'common cause triggered non-causal dependencies' (their pp. $26 \mathrm{ff}$.), i.e. that there is a common cause of the two correlated events (something which brings them into existence), but that the correlation is explained not in virtue of this common cause, but rather in terms of a nomological connection between them.

This idea can be put in the service of my dispositionalist account as follows: while the interaction with the environment causes the two systems' dispositions to manifest, the precise dynamics of states, amplitudes, and associated probabilities are then determined purely by the interaction Hamiltonian, i.e. nomologically. Which dispositions will manifest with what frequency in repeated trials is fixed by the Born rule, i.e. nomologically as well. In this sense the quantum correlations are triggered by a common cause (the environment) while being determined by laws of nature (the unitary dynamics and Born's rule, read in terms of propensities). So no 'spooky actions at a distance' (Einstein, 1947, p. 157; emphasis added) after all.

Notably, there is now no problem with special relativity on any level anymore, insofar as the relevant interaction (given by some Lagrangian) is Lorentz covariant: the correlation is ensured by a Lorentz covariant law and triggered by the dispositional profile of the joint quantum state. The state in turn acts as a common cause of there even being any measured values in the first place, which lies in the join of the past light cones of both detection events.

\section{Summary and Outlook}

In this paper I have provided an interpretation of QT and decoherence in terms of the manifestations of dispositions, thereby building on ideas that date back to Heisenberg's early interpretive efforts. My main suggestion was that decoherence should be understood in the sense of a mutual manifestation of dispositions in virtue of the interactions between the systems they pertain to. This manifestation would be gradual, in the sense of Hüttemann's CMDs, which allows that systems can be literally more or less localized, and have a more or less definite momentum at the same time. To read the formalism somewhat literally (and not introduce a collapse postulate after all), I introduced the notion of dominant manifestation, which would mean that all values of some observable would manifest on a system in consequence of a suitable interaction, but that only one of them would become amplified to such a degree that it would lead to observable behavior. The remainder I referred to as effectively latent. The Born rule-weights I took to quantify the strength of a disposition's tendency to become dominantly manifest. One might think of this in terms of a resource ('actuality') for which the values compete, in such a way that shares will be distributed unequally. ${ }^{15}$

My account has several merits: Unlike some other realist interpretations, it appreciates the

\footnotetext{
${ }^{15}$ Viewed by these lights, this suggests a way to make quite literal sense of Zurek's (2009) notion of 'Quantum Darwinism'.
} 
importance of decoherence right away; unlike the MWI it appreciates the central role of the Born rule right away, but does not have to reserve a special role for consciousness. It can make sense of observation, including such things as recoherence, while allowing that minds may supervene on brains and avoiding such things as mindless hulks.

However, some drawbacks or open questions remain: There is nothing in the formalism which corresponds directly to the selective amplification at stake; this is gathered rather from our successful practice of using the Born probabilities, and the experience of single outcomes. And there is the murky business of non-local influences, which haunts most realist interpretations. This can be dealt with by viewing the relation between the correlated outcomes as nomological, not causal; but strictly speaking, this in turn implies the need to provide an account of the laws of nature compatible with a non-causal reading.

I do not think that these are fatal flaws or insurmountable difficulties. Hence I suggest that a propensity-interpretation along the lines suggested above is an option that deserves more attention than it presently enjoys.

\section{Acknowledgments}

blinded for peer review

\section{References}

Albert, D. and Loewer, B. (1988). "Interpreting The Many Worlds Interpretation". Synthese, 77(2):195-213.

Arndt, M., Nairz, O., Petschinka, J., and Zeilinger, A. (2001). "High contrast interference with $\mathrm{C}_{60}$ and $\mathrm{C}_{70}$ ". Comptes Rendus de l'Académie des Sciences-Series IV-Physics, 2(4):581-585.

Baker, D. J. (2007). "Measurement outcomes and probability in Everettian quantum mechanics". Studies In History and Philosophy of Science Part B: Studies In History and Philosophy of Modern Physics, 38(1):153-169.

Bell, J. S. (1987[1964]). “On the Einstein-Podolsky-Rosen paradox". In Bell, J. S., editor, Speakable and unspeakable in quantum mechanics, pages 14-21. Cambridge, New York: Cambridge University Press.

Bell, J. S. (1990). "Against 'measurement'”. In Miller, A. I., editor, Sixty-Two Years of Uncertainty. Historical, Philosophical, and Physical Inquiries into the Foundations of Quantum Mechanics, pages 17-32. New York: Plenum Press.

Bohm, D. (1951). Quantum Theory. New York: Dover Publications Inc.

Brune, M., Hagley, E., Dreyer, J., Maitre, X., Maali, A., Wunderlich, C., Raimond, J., and Haroche, S. (1996). "Observing the Progressive Decoherence of the 'Meter' in a Quantum Measurement". Physical Review Letters, 77(24):4887.

Bub, J. (1997). Interpretating the Quantum World. Cambridge, New York: Cambridge University Press.

Carroll, S. M. and Sebens, C. T. (2014). "Many worlds, the born rule, and self-locating uncertainty”. In Struppa, D. C. and Tollaksen, J. M., editors, Quantum Theory: A Two-Time Success Story, pages 157-169. Springer. 
Colbeck, R. and Renner, R. (2012). "Is a System's Wave Function in One-to-One Correspondence with Its Elements of Reality?” Physical Review Letters, 108(15):150402(1-4).

d'Espagnat, B. (1990). “Towards a separable 'empirical reality'? Foundations of Physics, 20(10):1147-1172.

Dizadji-Bahmani, F. (2013). "The probability problem in Everettian quantum mechanics persists". The British fournal for the Philosophy of Science, 66(2):257-283.

Dorato, M. and Esfeld, M. (2010). "GRW as an Ontology of Dispositions". Studies in History and Philosophy of Science Part B: Studies in History and Philosophy of Modern Physics, 41(1):41-49.

Egg, M. and Esfeld, M. (2015). "Primitive ontology and quantum state in the GRW matter density theory”. Synthese, 192(10):3229-3245.

Einstein, A. (1947). Letter to born. In Born, M., editor, The Born-Einstein Letters, pages 157-160. London, Basingstoke: Macmillan.

Ellis, B. (2001). Scientific Essentialism. Cambrdige, New York: Cambridge University Press.

Ellis, B. and Lierse, C. (1994). "Dispositional Essentialism". Australasian fournal of Philosophy, 72(1):27-45.

Esfeld, M., Hubert, M., Lazarovici, D., and Dürr, D. (2014). "The Ontology of Bohmian Mechanics”. The British fournal for the Philosophy of Science, 65(4):773-796.

French, S. (2014). The Structure of the World: Metaphysics and Representation. Oxford University Press.

Friederich, S. (2013). "Interpreting Heisenberg interpreting quantum states". Philosophia Naturalis, 50(1):85-114.

Friedman, M. (1983). Foundations of Space-Time Theories. Relativistic Physics and Philosophy of Science. Princeton, New Jersey: Princeton University Press.

Fuchs, C. A. and Schack, R. (2012). "Bayesian Conditioning, the Reflection Principle, and Quantum Decoherence”. In Ben-Menahem, Y. and Hemmo, M., editors, Probability in physics, pages 233-248. Springer.

Gebharter, A. and Retzlaff, N. (2018). "A new proposal how to handle counterexamples to Markov causation à la Cartwright, or: Fixing the chemical factory". Synthese. https://doi.org/10.1007/s11229-018-02014-7.

Ghirardi, G., Marinatto, L., and Weber, T. (2002). "Entanglement and properties of Composite Quantum Systems: A Conceptual and Mathematical Analysis". Journal of Statistical Physics, 108(1-2):49-122.

Gillies, D. (2000). Philosophical Theories of Probability. London, New York: Routledge.

Hardy, L. (2004). "Quantum ontological excess baggage". Studies in History and Philosophy of Science Part B: Studies in History and Philosophy of Modern Physics, 35(2):267-276.

Hardy, L. (2013). “Are Quantum States Real?” International fournal of Modern Physics B, 27(13):1345012 (19 pp).

Healey, R. (2012). "Quantum Theory: a Pragmatist Approach". The British fournal for the Philosophy of Science, 63(4):729-771.

Heisenberg, W. (1971 [1958]). Physics and Philosophy. The Revolution in Modern Science. London: Allen \& Unwin Ltd., third edition.

Hüttemann, A. (2004). What's Wrong With Micro-Physicalism? London, New York: Routledge. Jaynes, E. T. (1990). "Probability in Quantum Theory". In Zurek, W. H., editor, Complexity, Entropy and the Physics of Information. Reading Massachussats: Addison-Wesley. 
Joos, E., Zeh, H., Kiefer, C., Giulini, D., Kupsch, J., and Stamatescu, I.-O. (2003). Decoherence and the Appearance of a Classical World in Quantum Theory. Berlin, Heidelberg: Springer, second edition.

Kent, A. (2010). "One World Versus Many: The Inadequacy of Everettian Accounts of Evolution, Probability, and Scientific Confirmation”. In Saunders, S., Barrett, J., Kent, A., and D., W., editors, Many Worlds? Everett, Quantum Theory, and Reality, pages 307-368. Oxford, New York: Oxford University Press.

Lockwood, M. (1996). “'Many Minds' Interpretations of Quantum Mechanics”. The British journal for the philosophy of science, 47(2):159-188.

Margenau, H. (1954). "Advantages and disadvantages of various interpretations of the quantum theory”. Journal of the Washington Academy of Sciences, 44(9):265-276.

Martin, C. B. (2008). The Mind in Nature. Oxford University Press.

Maudlin, T. (2014). "Critical Study: David Wallace, The Emergent Multiverse: Quantum Theory According to the Everett Interpretation”. Noûs, 48(4):794-808.

Mittelstaedt, P. (1998). "The Problem of Decoherence and the EPR Paradox". In Blanchard, P., Giulini, D., Joos, E., Kiefer, C., and Stamatescu, I.-O., editors, Decoherence: Theoretical, Experimental, and Conceptual Problems, pages 149-159. Berlin, Heidelberg: Springer.

Molnar, G. (2003). Powers: A Study in Metaphysics. Oxford University Press. Edited by Stephen Mumford.

Mumford, S. (1998). Dispositions. Oxford, New York: Oxford University Press.

Mumford, S. and Anjum, R. L. (2011). Getting Causes from Powers. Oxford University Press.

Näger, P. M. (2013). "A Stronger Bell Argument for Quantum Non-Locality". arXiv preprint arXiv:1308.3455.

Näger, P. M. (2016). “The causal problem of entanglement”. Synthese, 193(4):1127-1155.

Popper, K. R. (1957). "The Propensity Interpretation of the Calculus of Probability, and the Quantum Theory". In Körner, S., editor, Observation and Interpretation. A Symposium of Philosophers and Physicists. Proceedings of the Ninth Symposium of the Colston Research Society. London: Butterworths Scientific Publications.

Popper, K. R. (1995[1990]). A World of Propensities. Bristol: Thoemmes Press.

Pusey, M. F., Barrett, J., and Rudolph, T. (2012). "On the reality of the quantum state". Nature Physics, 8(6):475-478.

Raimond, J., Brune, M., and Haroche, S. (1997). "Reversible Decoherence of a Mesoscopic Superposition of Field States". Physical review letters, 79(11):1964.

Reichenbach, H. (1965). The Direction of Time. Mineloa, New York: Dover Publications, Inc. Edited by Maria Reichenbach.

Saunders, S., Barrett, J., Kent, A., and D., W., editors (2010). Many Words? Oxford, New York: Oxford University Press.

Schlosshauer, M. (2004). "Decoherence, the measurement problem, and interpretations of quantum mechanics". Reviews of Modern Physics, 76(4):1267-1305.

Schlosshauer, M. (2007). Decoherence and the Quantum to Classical Transition. Berlin, Heidelberg: Springer, second edition.

Spirtes, P., Glymour, C., and Scheines, R. (2000). Causation, Prediction, and Search. Cambridge, Massachusetts: MIT Press.

Suárez, M. (2004). "Causal processes and propensities in quantum mechanics ". Theoria, 
51(3):271-300.

Suárez, M. (2007). "Quantum propensities". Studies in History and Philosophy of Science Part B: Studies in History and Philosophy of Modern Physics, 38(2):418-438.

Suárez, M. (2009). "Propensities in Quantum Mechanics". In Greenberger, D., Hentschel, K., and Weinert, F., editors, Compendium of Quantum Physics. Concepts, Experiments, History and Philosophy, pages 502-5. Berlin, Heidelberg: Springer.

Taylor, E. F. and Wheeler, J. A. (1963). Spacetime Physics. San Francisco: W. H. Freeman and Company.

Vetter, B. (2015). Potentiality: From Dispositions to Modality. Oxford University Press.

von Mises, R. (1981 [1957]). Mathematical Theory of Probability and Statistics. New York: Dover Publications Inc., second revised edition.

Wallace, D. (2012). The Emergent Multiverse. Quantum Theory according to the Everett Interpretation. Oxford: Oxford University Press.

Wigner, E. (1983 [1961]). "Remarks on the mind-body question". In Wheeler, J. A. and Zurek, W. H., editors, Quantum Theory and Measurement, pages 168-181. Oxford, New York: Oxford University Press. Originally published in The Scientist Speculates, I. J. Good, ed., pp. 284-302, London: Heinemann.

Wigner, E. (1997 [1986]). “Are Quantum Mechanics and Relativity Theory Consistent?". In Wightman, A., editor, The Collected Works of Eugene Paul Wigner Part A, pages 374-379. Berlin, Heidelberg: Springer.

Wroński, L. (2014). Reichenbach's Paradise. Constructing the Realm of Probabilistic Common "Causes". Warsaw, Berlin: De Gruyter Open.

Zeh, H. (2000). "The Problem of Conscious Observation in Quantum Mechanical Description". Foundations of Physics Letters, 13(3):221-233.

Zeh, H. D. (1970). "On the Interpretation of Measurement in Quantum Theory". Foundations of Physics, 1(1):69-76.

Zurek, W. H. (1982). "Environment-Induced Superselection Rules". Physical Review D, $26(8): 1862$.

Zurek, W. H. (2005). "Probabilities from entanglement, Born's rule $p_{k}=\left|\psi_{k}\right|^{2}$ from envariance". Physical Review A, 71(5):052105.

Zurek, W. H. (2009). "Quantum darwinism”. Nature Physics, 5(3):181-188. 